Review

\title{
Efficacy and safety of gemcitabine plus anti-angiogenesis therapy for advanced pancreatic cancer: a systematic review and meta-analysis of clinical randomized phase III trials
}

\author{
Mengting Tong1,2\#, Jing Wang1\#, Hongliang Zhang2, Haibo Xing³, Yanling Wang1, Yong Fang1, Hongming \\ $\mathrm{Pan}^{1 凶}, \mathrm{Da} \mathrm{Li}^{1 凶}$ \\ 1. Department of Medical Oncology, Sir Run Run Shaw Hospital, Zhejiang University School of Medicine, 3\#, Eastern Qingchun Road, Jianggan District, \\ Hangzhou, Zhejiang, China, 310016 \\ 2. Second Department of Medical Oncology, The Fourth Affiliated Hospital of Xinjiang Medical University, 116\#, Huang He Road, Saybagh District, Urumqi, \\ Xinjiang, China, 830000 \\ 3. Intensive Care Department, Xiasha Campus, Sir Run Run Shaw Hospital, Zhejiang University, School of Medicine, 368\#, Xiasha Road, Jianggan District, \\ Hangzhou, Zhejiang, China, 310000
}

\#These authors contributed equally to this work.

$\square$ Corresponding authors: Hongming Pan, E-mail: hongming.pan@aliyun.com, Tel: +86-13605716662. Da Li, E-mail: lidaonconew@zju.edu.cn, Tel: $+86-13656710929$

(c) Ivyspring International Publisher. This is an open access article distributed under the terms of the Creative Commons Attribution (CC BY-NC) license (https://creativecommons.org/licenses/by-nc/4.0/). See http://ivyspring.com/terms for full terms and conditions.

Received: 2018.04.14; Accepted: 2018.10.28; Published: 2019.01.29

\begin{abstract}
Purpose: Pancreatic cancer is a common digestive neoplasm with a high fatality rate. We performed this systematic review and meta-analysis of clinical randomized phase III trials to explore the efficacy and safety of gemcitabine plus anti-angiogenesis therapy versus gemcitabine monotherapy for locally advanced or metastatic pancreatic cancer.

Methods: We searched PubMed, Embase and the Cochrane Library to identify eligible studies. Data were collected for the period from January 1, 2000 to August 20, 2018. Hazard ratios (HRs) and odds ratios (ORs) were used as main evaluation parameters.

Results: A total of eight eligible studies with 3,586 individuals were included in the present meta-analysis. The results showed that the combination of gemcitabine plus anti-angiogenesis therapy had a significant effect on progression-free survival $(\mathrm{HR}=0.92,95 \% \mathrm{Cl}: 0.86-1.00, P=0.04)$, but led to no significant difference in the overall survival $(\mathrm{HR}=0.96,95 \% \mathrm{Cl}: 0.88-1.05, P=0.38)$. In terms of safety, gemcitabine plus anti-angiogenesis therapy did not increase the rate of grade 3-4 common adverse effects except for hypertension.

Conclusions: Although gemcitabine plus anti-angiogenesis therapy might prolong the progression-free survival in locally advanced or metastatic pancreatic cancer, these successful results did not translate into a significant improvement in the overall survival or change in the clinical guidelines.
\end{abstract}

Key words: pancreatic cancer, anti-angiogenesis therapy, gemcitabine, meta-analysis

\section{Introduction}

Pancreatic cancer is a familiar digestive neoplasm with a high fatality rate that is the seventh most common cause of cancer-related deaths in the world [1]. Due to the challenging difficulties of early diagnosis of pancreatic cancer, almost $90 \%$ of the patients are diagnosed at a locally advanced or metastatic stage with a poorer prognosis [2]. In spite of the administration of combinations of traditional cytotoxic drugs, the development of targeted therapy, and the application of anti-angiogenesis therapy and even immunotherapy in the preceding decades, the 5 -year survival rate of pancreatic cancer patients has remained lower than those of cancers at other sites. Thus, pancreatic cancer has been considered as a 
chemoresistant malignant tumor. Currently, gemcitabine or capecitabine is considered as the keystone of the first-line chemotherapy for locally advanced or metastatic pancreatic cancer according to the latest new version of the National Comprehensive Cancer Network (NCCN) guideline [3].

In 1975, Folkman was the first to report that solid tumors needed high level of nutrition and oxygen supply during the processes of occurrence, development, and metastasis [4]. Now, anti-angiogenesis therapy has become a hot spot in the field of cancer treatment. The investigations on the application of anti-angiogenesis therapy for cancer treatment are mainly concentrated on drugs that target the vascular endothelial growth factor (VEGF) [5], the human epidermal growth factor receptor (EGFR/Her) family [6], and the phosphatidylinositol-3-kinase/ protein kinase/ mammalian target of rapamycin (PI3K/Akt/mTOR) signaling pathway [7], as well as on the efficacy of some traditional drugs, including matrix metalloproteinase inhibitors (MMPs) [8], and cyclooxygenase-2 (Cox-2) inhibitors [9]. Among those anti-angiogenesis drugs, agents which target the VEGF signaling pathway include the anti-VEGF monoclonal antibody, anti-VEGFR drugs, and soluble recombinant fusion proteins [10]. Numerous studies have demonstrated that traditional chemotherapy in combination with anti-angiogenesis therapy improves the overall survival of non-small cell lung cancer, colorectal cancer, ovarian cancer, etc.[5]. However, in the treatment of pancreatic cancer, the majority of anti-angiogenesis drugs have shown no great prospect in phase II/III clinical trials, and only erlotinib successfully completed phase III clinical trials [11]. Everolimus targets PI3K/Akt/mTOR signaling pathways and has also shown a remarkable potential in phase II clinical trials [12], but clinical phase III trials have not yet been conducted.

Therefore, this meta-analysis of previous clinical phase III trials was performed to evaluate the efficacy and safety of gemcitabine plus anti-angiogenesis versus gemcitabine monotherapy for locally advanced or metastatic pancreatic cancer.

\section{Methods}

This meta-analysis was conducted in accordance with the Preferred Reporting Items for Systematic Reviews and Meta-Analyses (PRISMA) guideline [13].

\section{Search strategy}

We searched PubMed, Embase, and the Cochrane Central Register of Controlled Trials electronic databases to identify eligible studies. The search data were collected for the period from January
1, 2000 to August 20, 2018. The following retrieval words were used: pancreas, pancreatic, and gemcitabine. In addition, we also checked the reference sections of the related reviews and meta-analyses to look for further reports.

\section{Inclusion and exclusion criteria}

All eligible studies were randomized phase III trials, which were required to evaluate the efficacy and safety of gemcitabine plus anti-angiogenesis versus gemcitabine monotherapy as first-line chemotherapy for the treatment of locally advanced or metastatic pancreatic cancer. Hence, by reading titles and abstracts, we excluded some phase I/II trails and studies which assessed the efficacy and safety of gemcitabine mono/double-therapy versus other targeted drugs or traditional radiochemotherapy.

\section{Data extraction}

The extracted information included:(1) the title of the paper, the name of the first author, and the publication year; (2) number of patients enrolled and treatment regimen in each arm, patient age, and gender; (3) accrual time, follow-up time, use of placebo and blinding, PS ECOG, disease stage; (4) median survival, $\mathrm{HRs}$ and $95 \% \mathrm{CI}$ of overall survival and progression-free survival, disease control rate and objective response rate; (5) common adverse events, including anemia, neutropenia, thrombocytopenia and hypertension. All data were cross-checked by two researchers.

\section{Quality assessment}

Two researchers assessed the quality of the individual studies independently by using Review Manager software (version 5.3, Cochrane Collaboration, Copenhagen, Denmark). Based in the results of a previous meta-analysis of RCTs, we evaluated the risk of the following types of bias in the randomized clinical trials included: detection bias, selection bias, reporting bias, performance bias, attrition bias and other potential bias [14].

\section{Statistical analysis}

HRs and 95\% CI were used to evaluate the therapeutic efficacy of the treatment of gemcitabine plus anti-angiogenesis therapy on the survival of pancreatic cancer patients. ORs and 95\% CI were utilized to estimate disease control rate, objective response rate, and the safety. Thus, the primary endpoint of this meta-analysis was efficacy, including overall survival, progression-free survival, disease control rate, and objective response rate. The rates of grade 3-4 adverse effects, which included anemia, neutropenia, thrombocytopenia, and hypertension, were regarded as secondary endpoints. All criteria 
were obtained directly from research or extracted from Kaplan-Meier survival curves by utilizing the software Engauge Digitizer version 4.1 (http://sourceforge.net/projects/digitizer) and Jayne F Tierney table (http://www.biomedcentral.com/ content/supplementary/1745-6215-8-16-S1.xls). A random-effect model was applied for all pooled results. Heterogeneity was estimated by $I^{2} ; I^{2}<50 \%$ indicated low heterogeneity, whereas $I^{2} \geq 50 \%$ denoted substantial heterogeneity. If the pooled results had substantial heterogeneity, we conducted a meta-regression analysis and subgroup meta-analysis to investigate the possible sources of the heterogeneity among studies. To explore the stability of the present meta-analysis, we performed a sensitivity analysis by sequentially omitting individual studies. This meta-analysis was implemented using the Review Manager 5.2 and STATA software (version 14.0, StataCorp, TX, USA). $P$-values were two-tailed and p-values $<0.05$ were considered statistically significant. Finally, the publication bias was assessed by utilizing funnel plots and the Egger's or Begg's test.

\section{Results}

\section{Study selection}

A total of 1,210 relevant studies were identified by using the search strategy described in the section of materials and methods. After reviewing their titles and abstracts, 1,153 studies were excluded, and the 57 studies we selected were assessed by full-text reviewing. Finally, eight eligible studies were included in the present meta-analysis based on the inclusion and exclusion criteria (flow diagram in Figure 1). All studies evaluated the efficacy of gemcitabine plus anti-angiogenesis therapy in locally advanced or metastatic pancreatic cancer patients, and six studies among them reported the most common adverse effects, including anemia, neutropenia, thrombocytopenia, and hypertension.

\section{Basic characteristics of the eligible studies}

The basic characteristics of 3,586 individuals included in the eight eligible clinical phase III trials, which compared the efficacy and safety of gemcitabine plus anti-angiogenesis drugs versus gemcitabine monotherapy, are summarized in Table 1. In five of the selected studies, the VEGF signaling pathway was targeted using sorafenib [15], axitinib [16], elpamotide [17], aflibercept [18] and bevacizumab [19]; in two of the studies the EGFR family by the administration of erlotinib [11] and cetuximab [20]; and in one, the matrix metalloproteinase inhibitor marimastat [21]. All eligible studies were random, placebo-controlled trials, except for that of cetuximab.

Table 1. Basic characteristics for eight eligible studies.

\begin{tabular}{|c|c|c|c|c|c|c|c|c|c|c|c|}
\hline $\begin{array}{l}\text { First } \\
\text { author }\end{array}$ & Year & Arm & Placebo/Blinding & No. & $\begin{array}{l}\text { Accrual } \\
\text { time }\end{array}$ & $\begin{array}{l}\mathrm{mOS} \\
(\mathrm{y})\end{array}$ & $\begin{array}{l}\text { Median } \\
\text { age }(y)\end{array}$ & $\begin{array}{l}\text { Sex } \\
\text { (M/F) }\end{array}$ & $\begin{array}{l}\text { PS } \\
\text { ECOG }\end{array}$ & $\begin{array}{l}\text { Mechanism of } \\
\text { anti-angiogenesis }\end{array}$ & Treatment regimen \\
\hline \multirow[t]{2}{*}{$\begin{array}{l}\text { Goncalves } \\
\text { [13] }\end{array}$} & 2012 & G+Sorafenib & $\mathrm{Y} / \mathrm{Y}$ & 52 & $\begin{array}{l}\text { Dec } \\
\text { 2006-Sep }\end{array}$ & 3.8 & $61(42-85)$ & $30 / 22$ & $\begin{array}{l}0-1: 42 \\
2: 5\end{array}$ & VEGFR inhibitor & $\begin{array}{l}\text { Gem } 1,000 \mathrm{mg} / \mathrm{m}^{2} \text {, weekly for } 1^{\text {st }} \text { cycle ( } 7 \text { weeks), } \\
\text { then weekly } 3 \text { every } 4 \text { weeks for cycle } 2 \text { and } 3\end{array}$ \\
\hline & & G & & 52 & 2009 & 5.7 & $64(40-82)$ & $32 / 20$ & $\begin{array}{l}0-1: 48 \\
2: 3\end{array}$ & & Sorafenib or placebo $200 \mathrm{mg}$ twice daily \\
\hline \multirow{2}{*}{$\begin{array}{l}\text { Kindler } \\
{[14]}\end{array}$} & 2011 & G+Axitinib & $\mathrm{Y} / \mathrm{Y}$ & 314 & Jul & 8.5 & $61(34-84)$ & $191 / 123$ & $0-1: 309$ & VEGFR inhibitor & Gem $1,000 \mathrm{mg} / \mathrm{m}^{2}$, weekly 3 every 4 weeks \\
\hline & & G & & 316 & $\begin{array}{l}2007-\text {-Oct } \\
2008\end{array}$ & 8.3 & $62(35-89)$ & $188 / 128$ & 0-1:312 & & Axitinib or placebo $5 \mathrm{mg}$ twice daily \\
\hline \multirow[t]{2}{*}{$\begin{array}{l}\text { Yamane } \\
{[15]}\end{array}$} & 2015 & G+Elpamotide & $\mathrm{Y} / \mathrm{Y}$ & 100 & $\begin{array}{l}\text { Jan } \\
\text { 2009-Jan }\end{array}$ & NA & $\begin{array}{l}63.5 \\
(38-80)\end{array}$ & $62 / 38$ & 0-1:100 & VEGFR inhibitor & Gem $1,000 \mathrm{mg} / \mathrm{m}^{2}$, weekly 3 every 4 weeks \\
\hline & & G & & 53 & 2010 & NA & $65(36-80)$ & $31 / 22$ & $0-1: 53$ & & $\begin{array}{l}\text { elpamotide } 2.0 \mathrm{mg} / \mathrm{ml} / \text { body or placebo } \\
1.0 \mathrm{ml} / \text { body }\end{array}$ \\
\hline \multirow[t]{2}{*}{$\begin{array}{l}\text { Rouguer } \\
{[16]}\end{array}$} & 2013 & G+Aflibercept & $\mathrm{Y} / \mathrm{Y}$ & 271 & $\begin{array}{l}\text { Dec } \\
\text { 2007-Sep }\end{array}$ & 6.5 & $62(34-88)$ & $160 / 11$ & $\begin{array}{l}0-1: 254 \\
2: 17\end{array}$ & $\begin{array}{l}\text { soluble VEGFR } \\
\text { inhibitor }\end{array}$ & $\begin{array}{l}\text { Gem } 1,000 \mathrm{mg} / \mathrm{m}^{2} \text {, weekly for } 1^{\text {st }} \text { cycle ( } 4 \text { weeks), } \\
\text { then weekly } 3 \text { every } 4 \text { weeks }\end{array}$ \\
\hline & & G & & 275 & 2009 & 7.8 & $61(34-86)$ & $157 / 118$ & $\begin{array}{l}0-1: 256 \\
2: 19\end{array}$ & & $4 \mathrm{mg} / \mathrm{kg}$ once every 2 weeks or placebo \\
\hline \multirow[t]{2}{*}{$\begin{array}{l}\text { Kindler } \\
{[17]}\end{array}$} & 2010 & $\mathrm{G}+\mathrm{Bev}$ & NA/NA & 302 & $\begin{array}{l}\text { Jun } \\
\text { 2004-Apr }\end{array}$ & 5.8 & $\begin{array}{l}63.7 \\
(26-88)\end{array}$ & $175 / 127$ & $\begin{array}{l}0-1: 266 \\
2: 36\end{array}$ & VEGF inhibitor & Gem $1,000 \mathrm{mg} / \mathrm{m}^{2}$, weekly 3 every 4 weeks \\
\hline & & G & & 300 & 2006 & 5.9 & $65(35-83)$ & $153 / 147$ & $\begin{array}{l}0-1: 270 \\
2: 30\end{array}$ & & $\mathrm{BEV} 10 \mathrm{mg} / \mathrm{kg}$ once every 2 weeks or placebo \\
\hline \multirow[t]{2}{*}{$\begin{array}{l}\text { Moore } \\
{[11]}\end{array}$} & 2007 & G+Erlotinib & $\mathrm{Y} / \mathrm{Y}$ & 285 & $\begin{array}{l}\text { Oct } \\
\text { 2001-Jan }\end{array}$ & 6.24 & $\begin{array}{l}63.7 \\
(37.7-84.4)\end{array}$ & $136 / 149$ & $\begin{array}{l}0-1: 230 \\
2: 54\end{array}$ & EGFR inhibitor & $\begin{array}{l}\text { Gem } 1,000 \mathrm{mg} / \mathrm{m}^{2} \text {, weekly for } 1^{\text {st }} \text { cycle ( } 7 \text { weeks), } \\
\text { then weekly } 3 \text { every } 4 \text { weeks }\end{array}$ \\
\hline & & G & & 284 & 2003 & 5.91 & $\begin{array}{l}64 \\
(36.1-92.4)\end{array}$ & $162 / 22$ & $\begin{array}{l}0-1: 232 \\
2: 52\end{array}$ & & Erlotinib or placebo 100 or $150 \mathrm{mg} / \mathrm{d}$ \\
\hline \multirow[t]{2}{*}{ Philip [18] } & 2010 & G+Cetuximab & NA/NA & 372 & $\begin{array}{l}\text { Jan } \\
\text { 2004-Apr }\end{array}$ & 6.3 & 63.7 & $190 / 182$ & $\begin{array}{l}0-1: 324 \\
2: 48\end{array}$ & EGFR inhibitor & $\begin{array}{l}\text { Gem } 1,000 \mathrm{mg} / \mathrm{m}^{2} \text {, weekly for } 1^{\text {st }} \text { cycle ( } 7 \text { weeks), } \\
\text { then weekly } 3 \text { every } 4 \text { weeks }\end{array}$ \\
\hline & & G & & 371 & 2006 & 5.9 & 64.3 & $200 / 171$ & $\begin{array}{l}0-1: 323 \\
2: 48\end{array}$ & & $\begin{array}{l}\text { Cetuximab } 400 \mathrm{mg} / \mathrm{m}^{2} \text { on week } 1 \text {, then weekly } \\
250 \mathrm{mg} / \mathrm{m}^{2}\end{array}$ \\
\hline \multirow{2}{*}{$\begin{array}{l}\text { Bramhall } \\
{[19]}\end{array}$} & 2002 & G+Marimastat & $\mathrm{Y} / \mathrm{Y}$ & 120 & Sep & $165 d$ & $62(32-83)$ & $69 / 51$ & KPS & MMPs inhibitor & Gem $1,000 \mathrm{mg} / \mathrm{m}^{2}$ \\
\hline & & G & & 119 & $\begin{array}{l}\text { 1997-Apr } \\
1998\end{array}$ & $164 d$ & $62(37-85)$ & $71 / 48$ & KPS & & Marimastat $10 \mathrm{mg}$ twice daily or placebo \\
\hline
\end{tabular}

*M, male; F, female; No., patients number; mOS, median Overall survival; NA, not available; Y, Yes; y, year; G or Gem, gemcitabine; PS, performance status. 


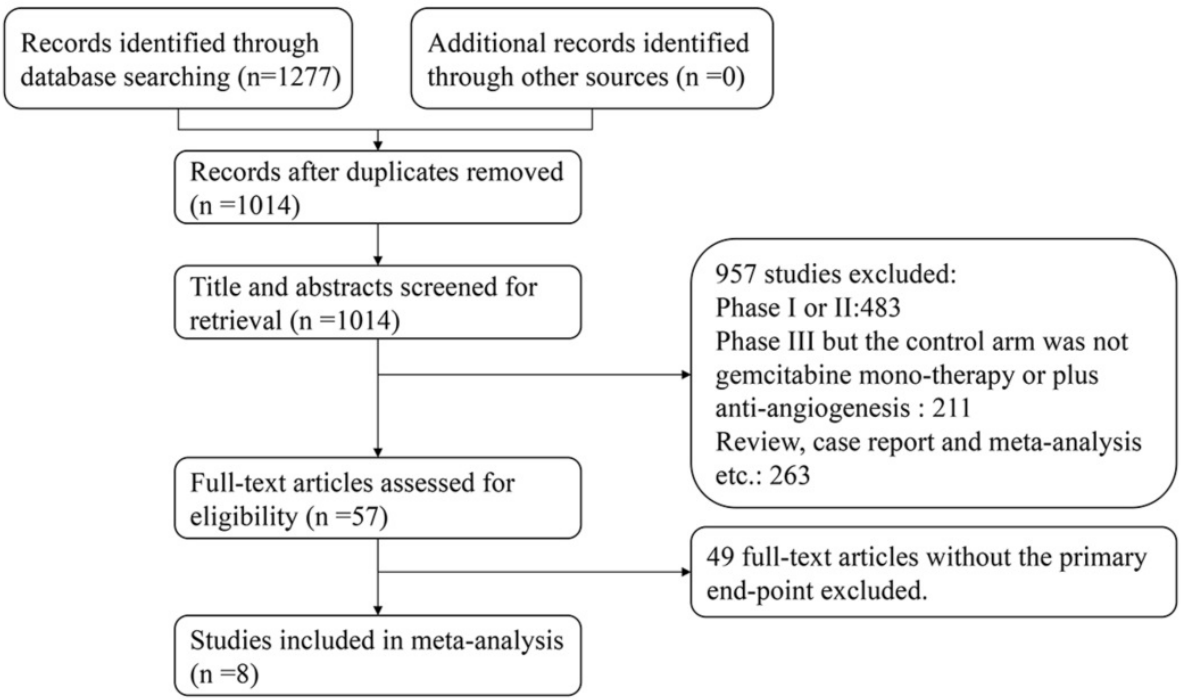

Figure 1. Flow diagram of the literature search

\section{Risk of bias in the individual trials}

The risks of bias in each included study were assessed according to the Cochrane reviewers' handbook (Figure 2). One study [20], which evaluated cetuximab, presented unclear risk in the blinding of participants and personnel as well as in the blinding of outcome assessment allocation concealment due to the absence of a random, placebo-controlled design.

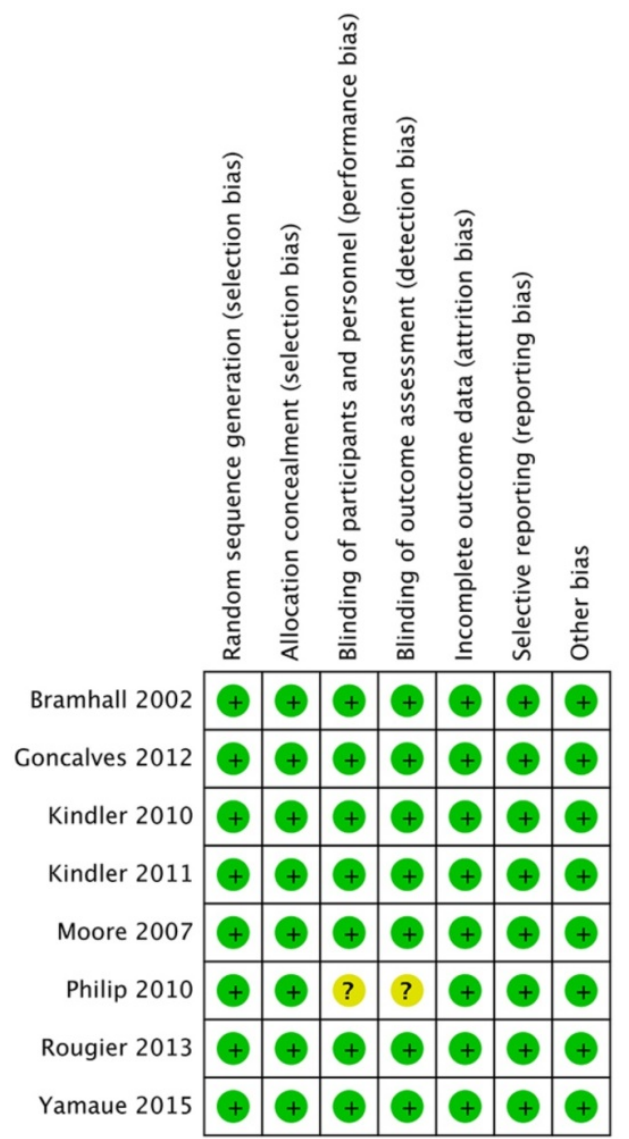

Figure 2. A summary table for the quality assessment of each study.

\section{Meta-analysis of primary endpoints}

\section{Overall survival}

The combined analysis of eight studies showed that there was no significant difference in the overall survival of locally advanced and metastatic pancreatic cancer patients between gemcitabine plus anti-angiogenesis therapy and gemcitabine monotherapy $(\mathrm{HR}=0.96,95 \% \mathrm{CI}: 0.88-1.05, P=0.38$, $\left.I^{2}=11 \%\right)($ Figure 3A).

\section{Progression-free survival}

A significant difference was found in the progression-free survival between gemcitabine plus anti-angiogenesis therapy and gemcitabine monotherapy $(\mathrm{HR}=0.92,95 \% \mathrm{CI}: 0.86-1.00, P=0.04)$ without significant heterogeneity $\left(I^{2}=0 \%\right)$ (Figure 3B).

\section{Disease control rate and objective control rate}

Post-treatment disease control rate (DCR) was determined in seven studies. Using the random-effect model, we found no significant differences between the two different therapy groups (OR $=0.99,95 \% \mathrm{CI}$ : $0.77-1.28, P=0.96$ ) (Figure $3 C$ ). Five studies reported the objective response rate (ORR) and established no significant improvement after the application of gemcitabine plus anti-angiogenesis therapy $(\mathrm{OR}=$ 1.26, 95\% CI: $0.90-1.77, P=0.18$ ) (Figure 3D).

\section{Meta-analysis of secondary endpoints}

A total of six studies reported the rate of grade 3-4 adverse effects, among which, five included anemia, six neutropenia, five thrombocytopenia, and three hypertension. All results were also combined by the random-effect model. No significant difference between gemcitabine plus anti-angiogenesis therapy 
and gemcitabine monotherapy was detected for the cases of anemia (OR $=0.71,95 \% \mathrm{CI}$ : $0.30-1.70, P=$ 0.44 ) (Figure $4 \mathrm{~A})$, neutropenia $(\mathrm{OR}=1.00,95 \% \mathrm{CI}: 0.59$ - 1.68, $P=1.00$ ) (Figure $4 \mathrm{~B}$ ), and thrombocytopenia $(\mathrm{OR}=1.43,95 \% \mathrm{CI}: 0.83-2.47, P=0.20)$ (Figure $4 \mathrm{C})$.
Moreover, gemcitabine plus anti-angiogenesis therapy significantly increases the incidence of the patients with hypertension (OR $=4.11,95 \% \mathrm{CI}$ : 1.95 $8.64, P=0.0002$ ) (Figure $4 \mathrm{D})$.

A. OS

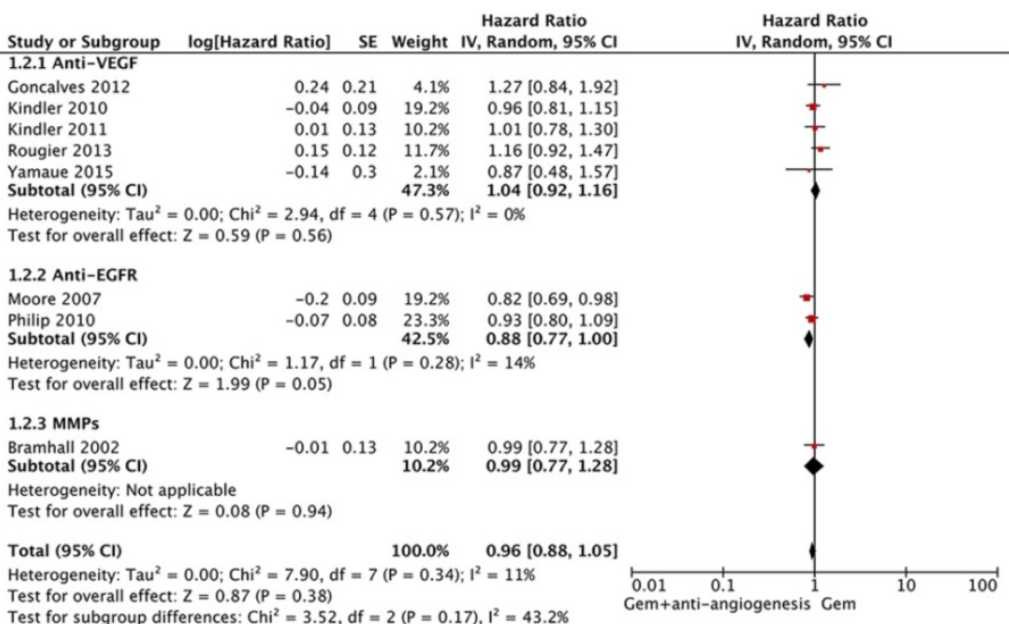

B. PFS

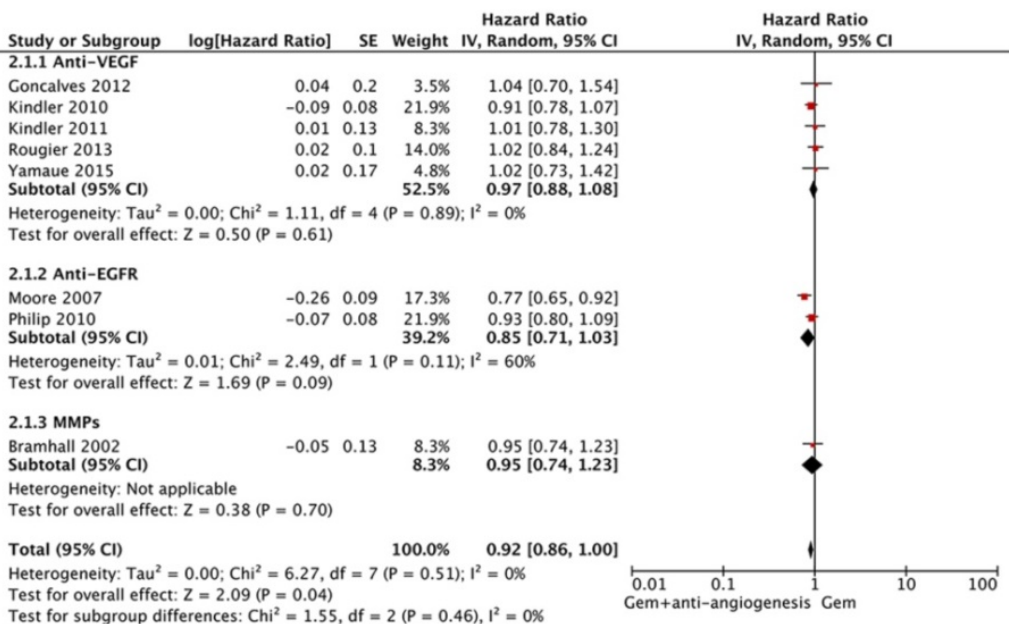

C. DCR

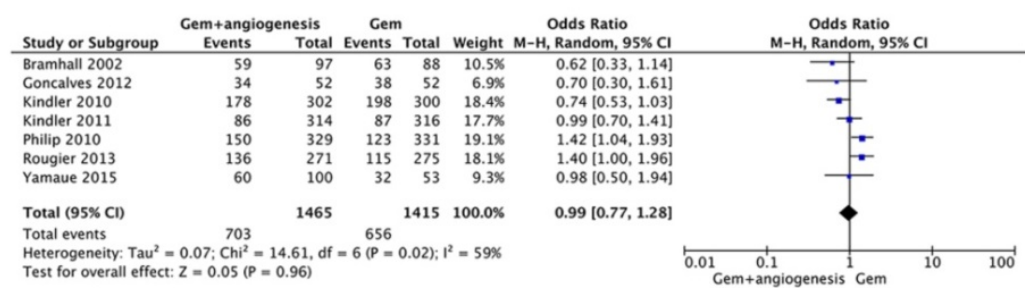

D. ORR

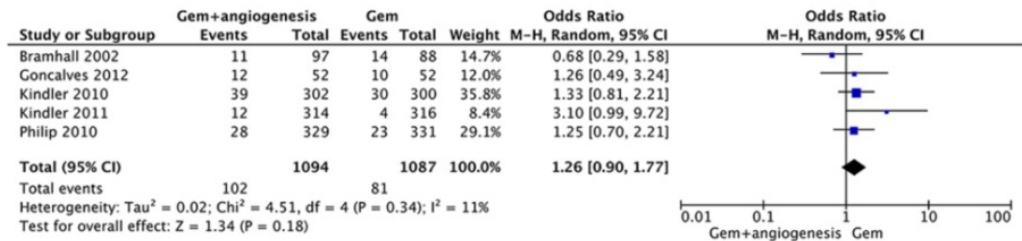

Figure 3. Meta-analysis of the primary endpoints. The forest plot for efficacy: (A) Overall survival; (B) Progression-free survival; (C) Disease control rate; (D) Objective response rate. 
A. anemia

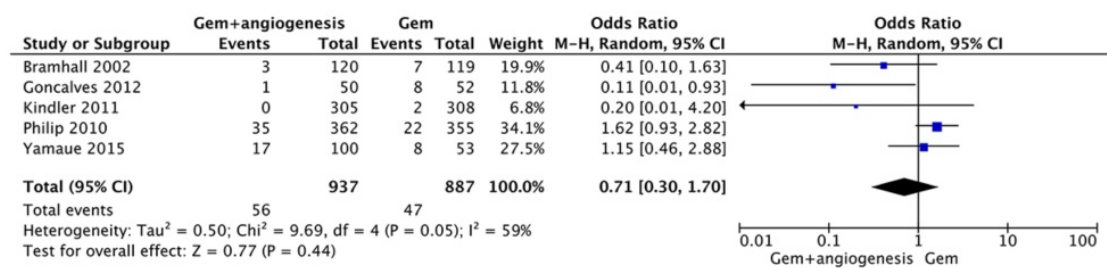

\section{B. neutropenia}

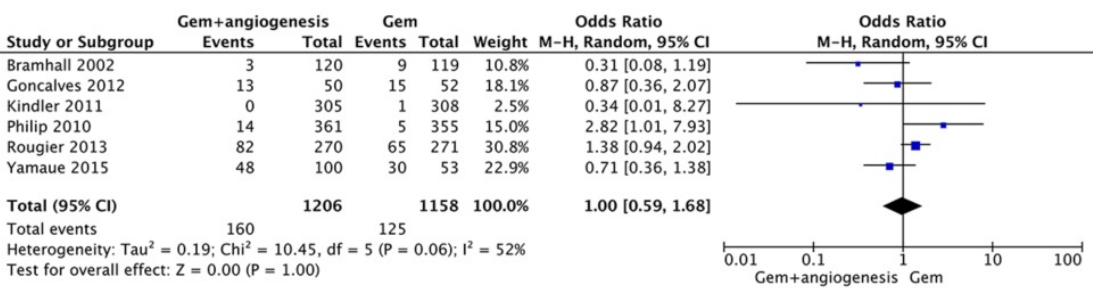

\section{C. thrombocytopenia}

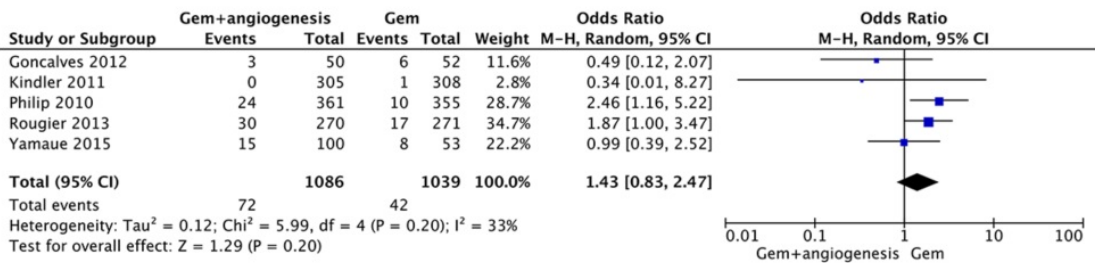

\section{D.hypertension}

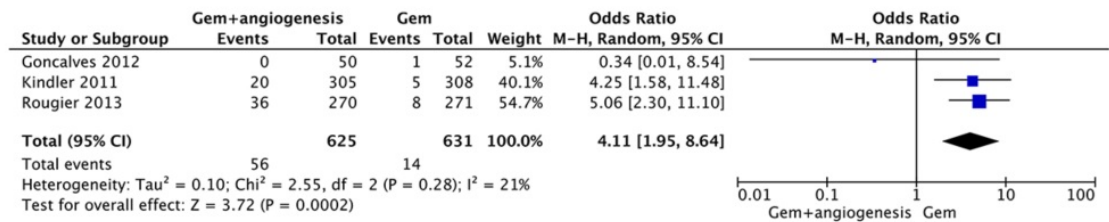

Figure 4. Second endpoints. The forest plot for grade 3-4 adverse effects rate: (A) Anemia; (B) Neutropenia; (C) Thrombocytopenia; (D) Hypertension

\section{Sensitivity analysis}

We excluded individual studies in turn to explore the stability of this meta-analysis. No significant differences were observed except for the pooled HR of the progression-free survival (Table 2).

Table 2. Sensitive analysis for progression free survival

\begin{tabular}{lllll}
\hline First author & Publication year & HRs & $95 \%$ CI & $P$-value \\
\hline Goncalves [13] & 2012 & 0.92 & $0.85-0.99$ & 0.03 \\
Kindler [17] & 2010 & 0.93 & $0.85-1.01$ & 0.09 \\
Kindler [14] & 2011 & 0.92 & $0.85-0.99$ & 0.03 \\
Rouguer [16] & 2013 & 0.91 & $0.84-0.99$ & 0.02 \\
Yamaue [15] & 2015 & 0.92 & $0.85-0.99$ & 0.03 \\
Moore [11] & 2007 & 0.96 & $0.89-1.04$ & 0.33 \\
Philip [18] & 2010 & 0.92 & $0.85-1.01$ & 0.07 \\
Bramhall [19] & 2002 & 0.92 & $0.85-1.00$ & 0.05
\end{tabular}

\section{Publication bias}

The funnel plot of HRs of the overall survival was symmetric (Figure 5A), and the publication bias assessed by the Egger's or Begg's test was also not significant $(P=0.250, P=0.454$, respectively $)$.
Symmetry in the funnel plot was also observed for the progression-free survival (Figure 5B), and the Egger's or Begg's test results showed no significant potential publication bias $(P=0.386, P=0.283$, respectively).

\section{Discussion}

Pancreatic cancer is regarded as a chemoresistant neoplasm with a poor prognosis. Previous studies attempted to explore many new anticancer drugs or chemotherapy treatments. However, most of them did not obtain satisfactory results. With the rapid introduction of a large number of novel anti-angiogenesis drugs in the past decade, only the combination of gemcitabine and erlotinib improved the overall survival by two weeks for pancreatic cancer patients [11].

Angiogenesis which regulates mainly by VEGF signaling pathway and EGFR family has an important role in the development and metastasis of tumors. Many studies have revealed that intracellular tyrosine phosphorylation of VEGFR is activated after the combination of VEGF and VEGFR and then promotes 


\section{A. Funnel plot for OS}

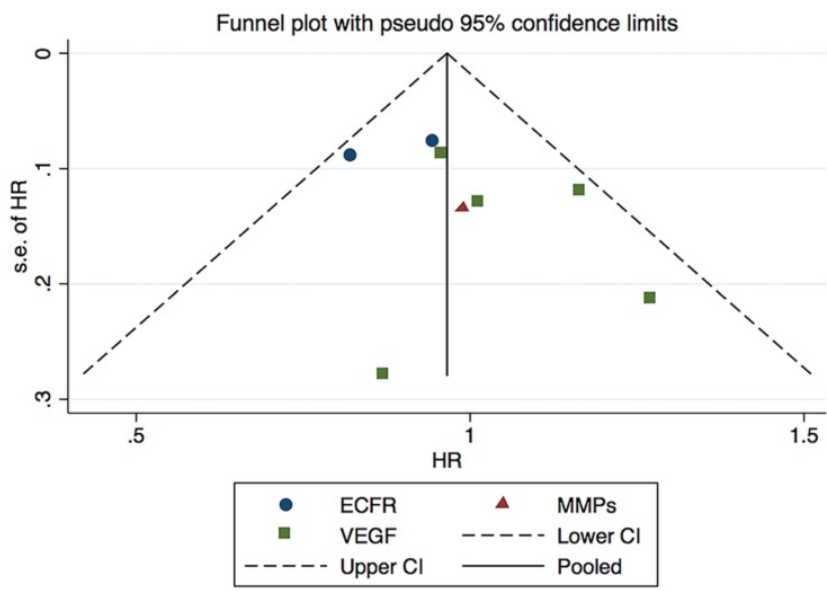

\section{B. Funnel plot for PFS}

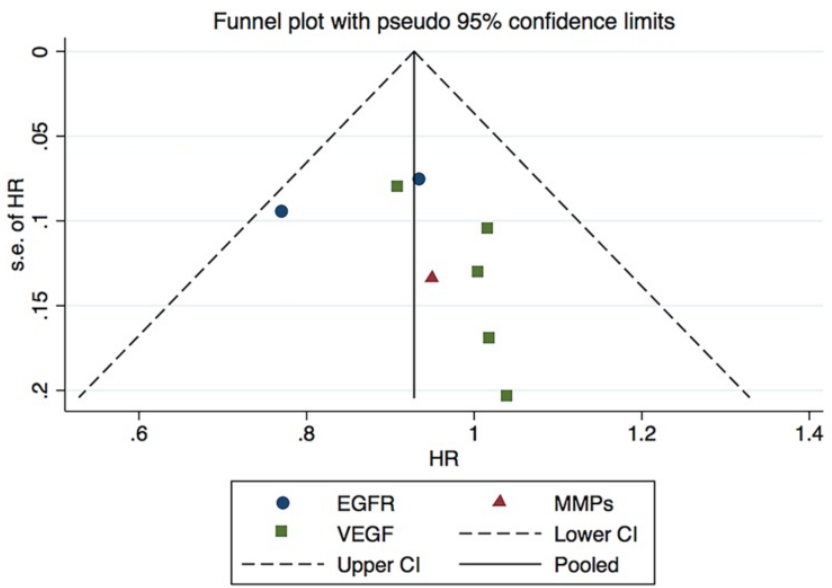

Figure 5. Funnel plot of the meta-analysis on the overall survival (A) and progression-free survival (B)

the angiogenesis of tumors. The EGFR family has 4 members, namely HER-1(also known as EGFR, $40 \%-80 \%$ of pancreatic cancer patients with EGFR overexpression.), ErbB-2(meaning Her-2), ErbB-3 and ErbB-4. Some basic researches also have shown that anti-angiogenesis drugs for EGFR family could suppress tumor growth and metastasis by inhibiting tumor angiogenesis.

Bevacizumab is a recombinant human monoclonal IgG1 antibody which targeted VEGF-A and also the first antibody to be approved to gain great achievement in non-small cell lung cancer, colon cancer and cervical cancer et al. in the United States. However, exploration of bevacizumab in pancreatic cancer is still in CALGB 80303 study [19]. Compared with gemcitabine monotherapy, progression-free survival in group of gemcitabine plus Bevacizumab extended for 0.9 months ( 3.8 vs. 2.9 months), but the benefit of progression-free survival did not translate into benefit of overall survival (5.8 vs.5.9 months). Moreover, there was a significant increase in adverse events such as proteinuria and hypertension in patients treated with combination therapy.
Tyrosine kinase inhibitors (TKI) include sorafenib, sunitinib, axitinib and vandetanib which inhibit VEGFR-1, VEGFR-2 and VEGFR-3, as well as elpamotide targeting single VEGFR-2. BAYPAN study with 104 advanced pancreatic cancer patients showed that gemcitabine plus sorafenib compared with gemcitabine monotherapy did not improve overall survival (8 vs.9.2 months) [15]. A clinical phase II trial of gemcitabine plus sorafenib versus sorafenib obtained similar results at the same time [22]. So, we could draw a preliminary conclusion that pancreatic cancer patients did not benefit from sorafenib. GISCAD study also confirmed this conclusion in 2014, the response rate of gemcitabine and cisplatin combined with sorafenib was only $3.4 \%$ [23]. Like sorafenib, sunitinib also has suffered many setbacks. Progression-free survival was shortened to 11.6 weeks in combination group with neutrophils decreased significantly compared with 13.3 weeks of gemcitabine monotherapy in CESADR study [24]. For axitinib, there was also no statistically significant difference in overall survival but with higher severe adverse reactions in AG-013736 study [16]. Significant 
survival benefits were not observed in gemcitabine combined with elpamotide similarly [17].

Previous studies have demonstrated that pancreatic cancer patients could not benefit from anti-VEGF signaling pathway therapy. Gemcitabine combined with vandetanib confirmed this conclusion again in a clinical phase II trial recently [25]. Apatinib with highly selective targeted VEGFR-2 is a self-developed TKI in our country and also has been approved for advanced gastric cancer. We expect the result of OECTAPGAMPC study which explores the efficiency and safety of gemcitabine plus apatinib. Ramucirumab, a representative monoclonal antibody against VEGFR-2, as well as combined paclitaxel can improve OS of gastric cancer significantly. Although there are no important results for pancreatic cancer, we look forward to the results of NCT02581215 (mFOLFIRINOX \pm Ramucirumab). In addition, aflibercept, a soluble antibody, is a fusion protein of VEGFR extracellular regions and the human immunoglobulin $\mathrm{G}$ constant region. A clinical phase III trial showed pancreatic cancer patients could not also benefit from gemcitabine combined with aflibercept [18].

In general, anti-angiogenesis drugs against VEGF signaling pathways do not possess the significant effects in the treatment of pancreatic cancer.

Anti-angiogenesis drugs against VEGF signaling pathways consist of two categories. One is a small molecule compound which can inhibit tyrosine kinase activity by combining with EGFR intracellular segment to block G1 period in tumor cell. The other is a monoclonal antibody. The specific conjugation of anti-angiogenesis antibodies and cell membrane receptors inhibits the proliferation of tumor cells by interfering with EGFR phosphorylation and hindering the dimer formation [26].

The representative drug of the small molecule compound is erlotinib. NCICPA.3 study included 569 locally advanced or distant metastasis pancreatic cancer patients showed that gemcitabine combined with erlotinib could significantly improve progression-free survival and overall survival. Despite the fact that median overall survival was only extended by 2 weeks (6.24 and 5.91 months), the one-year survival rate increased significantly to $23 \%$ from $17 \%(p=0.038)$ [11]. AIO-PK0104 trial further found that patients with k-ras mutation might benefit more from erlotinib [27]. Some scholars also explored the difference of capecitabine and gemcitabine conjunction with the erlotinib based on XELTA study [28]. It is expected that there are no significant differences in efficacy but with higher haematological toxicity in capecitabine group $[29,30]$.
Cetuximab, a representative anti-angiogenesis monoclonal antibody against EGFR, is a human rat chimeric type IgG1 antibody. S0205 study showed that, compared with gemcitabine monotherapy, gemcitabine plus cetuximab could only prolong overall survival to 6.3 months from 5.9 months without significant statistical significance [20]. Results of the ATIP study announced in 2013, gemcitabine plus cetuximab used for postoperative adjuvant therapy in pancreatic cancer patients also did not gain significant survival benefits, and 18-months' objective disease response rate (ORR) was only 27.1\% (ORR of gemcitabine monotherapy was 35\%). What's more, there is no satisfactory effect in patients with K-ras mutation in subgroup analysis [31]. Similarly, three drug combinations of oxaliplatin, gemcitabine plus cetuximab [32] or gemcitabine, bevacizumab combined with cetuximab [33] also did not achieve the desired effect. Fortunately, Esnaola et al. found that neoadjuvant chemotherapy of oxaliplatin, gemcitabine plus cetuximab could increase the $\mathrm{R} 0$ resection rate in some pancreatic cancer patients [34]. Although it was a clinical phase II trial with 37 patients evaluated, it indicated that treatment strategies might need to be changed for monoclonal antibodies against EGFR used in the treatment of pancreatic cancer.

Trastuzumab is a recombinant DNA derived human monoclonal IgGl antibody and also is the representative drug of Her-2 inhibitors which have gained great achievement in breast cancer as well as gastric cancer with Her-2 overexpression. A clinical phase II trial reported that capecitabine combined with trastuzumab could not improve progression-free survival and overall survival of pancreatic cancer patients with Her-2 overexpression [35]. Her-3 with no kinase activity is usually as a dimer combined with Her-2. Pertuzumab can hinder the dimer formation of Her-2 oneself and the heterogeneous dimer of Her-2 and Her-3. A study suggested that Her-3 might be a biomarker for the treatment of pertuzumab but with rare clinical data [36]. Lapatinib is a double inhibitor for EGFR and Her-2. An one-arm phase II clinical trial of gemcitabine combined with lapatinib also failed with only 4 months survival period in the first-line treatment of pancreatic cancer [37]. However, this combination obtained relatively satisfactory results in the second-line treatment with a survival period of 5.2 months [38].

Other anti-angiogenesis drugs include PI3K/Akt/mTOR signaling pathway inhibitors, MMPs inhibitors, Cox-2 inhibitors, fibroblast growth factor inhibitors (FGF), platelet derived growth factor (PDGF) inhibitors, and endostatin. Everolimus, a representative mTOR inhibitor, can block tumor 
angiogenesis by interfering VEGF signaling pathway. A phase II trial showed that the oral medicines of capecitabine plus everolimus might have survival benefits with progression-free survival of 3.6 months and overall survival of 8.9 months for patients with advanced pancreatic cancer [12]. In addition to everolimus, antitumor mechanism of other anti-angiogenesis mentioned above has not yet been fully understood at present, it is regretful that each clinical trial draws different conclusions and many researches are phase II trials with small sample.

Progression-free survival is a credible endpoint especially in good designed and blinded trials. The advantages of progression-free survival are that it appears much earlier than overall survival without the potential affection of subsequent treatment and also can reflect the growth of the tumor (this phenomenon may reflect the tumor related illness or death). Unlike progression-free survival, overall survival, another common endpoint, influences by many factors in the full treatment of cancer patients. For instance, follow up treatment after disease progression, quality of life. This systematic review and meta-analysis demonstrates that gemcitabine plus anti-angiogenesis therapy seems to prolong the progression-free survival despite the instability of the results. Unfortunately, no significant improvement was established in the overall survival. These outcomes are also exceedingly common in other malignant tumors [39]. Thus, it is necessary to elucidate the reason for the absence of positive effects on the overall survival in the treatment of pancreatic cancer patients with anti-angiogenesis agents in spite of the successfully achieved prolongation of the progression-free survival.

The findings of the present and previous fundamental studies have indicated that, in contrast to other cancer types, pancreatic cancer might not be responsive to anti-angiogenesis therapy. Many scholars considered that the poor density of the vessels embedded in the stroma is a specific characteristic that could contribute to limitation of drug delivery [40] and reduction in anti-angiogenesis drug diffusion [41]. Other potential mechanisms of drug-resistance have also been reported. For example, some authors have suggested that pancreatic tumors become more invasive and continue to grow by increasing their capabilities for invasion without angiogenesis. Based on this concept, Casanovas et al. found a pro-invasive adaptation mechanism in a pancreatic tumor model in their study assessing the effects of VEGFR inhibitors [42]. In addition, Xian et al. demonstrated that the integrality of pericyte coverage of tumor vessels limited metastasis in a model of pancreatic tumor [43]. Further research has also revealed that the expression of PDGFR-B in the pancreatic tumor induces pericyte recruitment during therapy with a combination of anti-VEGF inhibitors [44]. Furthermore, cancer stem cells (CSCs), which are regarded as highly chemoresistant cells with metastatic potential, have been detected in pancreatic cancer. In addition, chemoresistance may be related to high molecular heterogeneity of pancreatic cancer [45]. Therefore, it can be concluded that the reasons for natural drug resistance in pancreatic cancer have not been elucidated. But we guess, this might be a good option for pancreatic cancer patients that intra-tumor injection of anti-angiogenesis drugs or chemotherapy drugs which can break the blood-pancreatic barrier and increase drug concentration within the tumor with fewer side effects.

Numerous previous numerous meta-analyses were also conducted to explore the efficacy of the combination of gemcitabine and targeted therapy or erlotinib alone [46] in pancreatic cancer treatment. However, the control arm of the meta-analysis included only few anti-angiogenesis drugs, and generally, the data were obtained from clinical phase II/III trials and some prognostic/biomarker identification studies performed in the past decade [47]. Our meta-analysis was conducted to pool all clinical phase III trials of gemcitabine plus anti-angiogenesis therapy in locally advanced or metastatic pancreatic cancer. Thus, our report was not considered a subgroup analysis of the previous studies. Moreover, the emphasis of our research was on the pooled results including one matrix metalloproteinase inhibitor (marimastat). It is well known that matrix metalloproteinase inhibitors can inhibit the growth and metastases, and suppress tumor neovascularization [48]. Nevertheless, the overall survival of pancreatic cancer patients treated with marimastat was not significantly different from that of the patients treated with placebo [21].

These anti-angiogenesis drugs also share mechanism-related toxicities, of which the most frequent adverse effects are hypertension, proteinuria, thromboembolism, and fistula formation [10]. For clinical practice, it is important that those drugs have lower hematologic toxicity than traditional cytotoxic drugs. In this meta-analysis, we also found that the combination of gemcitabine and anti-angiogenesis drugs does not increase the rate of grade $3-4$ adverse effects, except for hypertension.

There are several limitations in our research. First, the present meta-analysis included only eight phase III trials with 3,586 individuals. Moreover, the control arms of the study assessed the efficacy of cetuximab without the use of placebo, as we 
considered that this design had no effects on the final combined results. Second, many studies did not provide valid data about adverse effects, such as, proteinuria, thromboembolism, and fistula formation. Finally, HRs and 95\% CI, which were extracted from Kaplan-Meier survival curves in the two studies, might have exerted potential influence on the pooled results.

In conclusion, despite the fact that the results of this meta-analysis do not change clinical guidelines, they may suggest the eventual designs of future trials. Thus, further research should focus on multi-target agents or applications of combinations of two or three anti-angiogenesis drugs, traditional cytotoxic drugs, and even immunomodulatory drugs in the treatment of pancreatic cancer.

\section{Acknowledgements}

\section{Funding}

The National Natural Science Foundation of China (81573003) and the Medical Science and Technology Project of Zhejiang Province (2015KYB217).

\section{Competing Interests}

The authors have declared that no competing interest exists.

\section{References}

1. Siegel RL, Miller KD, Jemal A. Cancer Statistics, 2017. CA: a cancer journal for clinicians. 2017;67(1):7-30. doi: 10.3322/caac.21387. PubMed PMID: 28055103.

2. Kamisawa T, Wood LD, Itoi T, Takaori K. Pancreatic cancer. Lancet (London, England). 2016;388(10039):73-85. doi: 10.1016/S0140-6736(16)00141-0. PubMed PMID: 26830752.

3. [Internet] NCCN guidelines for pancreatic cancer. Accessed 2017. Available from: http://www.nccn.org/professionals/physician_gls/pdf/pancreatic .pdf

4. Folkman J. Tumor angiogenesis: a possible control point in tumor growth Annals of internal medicine. 1975;82(1):96-100.

5. Ferrara N, Adamis AP. Ten years of anti-vascular endothelial growth factor therapy. Nat Rev Drug Discov. 2016;15(6):385-403.

6. Dancey JE, Freidlin B. Targeting epidermal growth factor receptor--are we missing the mark? Lancet (London, England). 2003;362(9377):62-4.

7. Falasca M, Selvaggi F, Buus R, Sulpizio S, Edling CE. Targeting phosphoinositide 3-kinase pathways in pancreatic cancer--from molecular signalling to clinical trials. Anticancer Agents Med Chem. 2011;11(5):455-63.

8. Wojtowicz-Praga SM, Dickson RB, Hawkins MJ. Matrix metalloproteinase inhibitors. Investigational new drugs. 1997;15(1):61-75.

9. Gately S, Li WW. Multiple roles of COX-2 in tumor angiogenesis: A target for antiangiogenic therapy. Seminars in Oncology. 2004;31(2):2-11.

10. Jayson GC, Kerbel R, Ellis LM, Harris AL. Antiangiogenic therapy in oncology: current status and future directions. Lancet (London, England). 2016;388(10043):518-29

11. Moore MJ, Goldstein D, Hamm J, Figer A, Hecht JR, Gallinger S, et al. Erlotinib plus gemcitabine compared with gemcitabine alone in patients with advanced pancreatic cancer: a phase III trial of the National Cancer Institute of Canada Clinical Trials Group. Journal of clinical oncology: official journal of the American Society of Clinical Oncology. 2007;25(15):1960-6.

12. Kordes S, Klumpen HJ, Weterman MJ, Schellens JH, Richel DJ, Wilmink JW. Phase II study of capecitabine and the oral mTOR inhibitor everolimus in patients with advanced pancreatic cancer. Cancer chemotherapy and pharmacology. 2015;75(6):1135-41.

13. Liberati A, Altman DG, Tetzlaff J, Mulrow C, Gotzsche PC, Ioannidis JP, et al. The PRISMA statement for reporting systematic reviews and meta-analyses of studies that evaluate healthcare interventions: explanation and elaboration. BMJ. 2009;339:b2700.

14. Shi L, Pu J, Xu L, Malaguit J, Zhang J, Chen S. The efficacy and safety of cilostazol for the secondary prevention of ischemic stroke in acute and chronic phases in Asian population--an updated meta-analysis. BMC Neurol. 2014;14:251.

15. Goncalves A, Gilabert M, Francois E, Dahan L, Perrier H, Lamy R, et al. BAYPAN study: a double-blind phase III randomized trial comparing gemcitabine plus sorafenib and gemcitabine plus placebo in patients with advanced pancreatic cancer. Annals of oncology: official journal of the European Society for Medical Oncology. 2012;23(11):2799-805.

16. Kindler HL, Ioka T, Richel DJ, Bennouna J, Letourneau R, Okusaka T, et al. Axitinib plus gemcitabine versus placebo plus gemcitabine in patients with advanced pancreatic adenocarcinoma: a double-blind randomised phase 3 study. The Lancet Oncology. 2011;12(3):256-62.

17. Yamaue H, Tsunoda T, Tani M, Miyazawa M, Yamao K, Mizuno N, et al. Randomized phase II/III clinical trial of elpamotide for patients with advanced pancreatic cancer: PEGASUS-PC Study. Cancer science. 2015;106(7):883-90

18. Rougier P, Riess H, Manges R, Karasek P, Humblet $Y$, Barone C, et al. Randomised, placebo-controlled, double-blind, parallel-group phase III study evaluating aflibercept in patients receiving first-line treatment with gemcitabine for metastatic pancreatic cancer. European journal of cancer (Oxford, England: 1990). 2013;49(12):2633-42.

19. Kindler HL, Niedzwiecki D, Hollis D, Sutherland S, Schrag D, Hurwitz H, et al. Gemcitabine plus bevacizumab compared with gemcitabine plus placebo in patients with advanced pancreatic cancer: phase III trial of the Cancer and Leukemia Group B (CALGB 80303). Journal of clinical oncology: official journal of the American Society of Clinical Oncology. 2010;28(22):3617-22.

20. Philip PA, Benedetti J, Corless CL, Wong R, O'Reilly EM, Flynn PJ, et al. Phase III study comparing gemcitabine plus cetuximab versus gemcitabine in patients with advanced pancreatic adenocarcinoma: Southwest Oncology Group-directed intergroup trial S0205. Journal of clinical oncology: official journal of the American Society of Clinical Oncology. 2010;28(22):3605-10.

21. Bramhall SR, Schulz J, Nemunaitis J, Brown PD, Baillet M, Buckels JA. A double-blind placebo-controlled, randomised study comparing gemcitabine and marimastat with gemcitabine and placebo as first line therapy in patients with advanced pancreatic cancer. British journal of cancer. 2002;87(2):161-7.

22. El-Khoueiry AB, Ramanathan RK, Yang DY, Zhang W, Shibata S, Wright JJ, et al. A randomized phase II of gemcitabine and sorafenib versus sorafenib alone in patients with metastatic pancreatic cancer. Investigational new drugs. 2012;30(3):1175-83.

23. Cascinu S, Berardi R, Sobrero A, Bidoli P, Labianca R, Siena S, et al. Sorafenib does not improve efficacy of chemotherapy in advanced pancreatic cancer: A GISCAD randomized phase II study. Digestive and liver disease: official journal of the Italian Society of Gastroenterology and the Italian Association for the Study of the Liver. 2014;46(2):182-6.

24. Bergmann L, Maute L, Heil G, Russel J, Weidmann E, Koberle D, et al. A prospective randomised phase-II trial with gemcitabine versus gemcitabine plus sunitinib in advanced pancreatic cancer: a study of the CESAR Central European Society for Anticancer Drug Research-EWIV. European journal of cancer (Oxford, England: 1990). 2015;51(1):27-36.

25. Middleton G, Palmer DH, Greenhalf W, Ghaneh P, Jackson R, Cox T, et al. Vandetanib plus gemcitabine versus placebo plus gemcitabine in locally advanced or metastatic pancreatic carcinoma (ViP): a prospective, randomised, double-blind, multicentre phase 2 trial. The Lancet Oncology. 2017; 18: 486-99.

26. Li E, et al. Tumor targeted therapy. [M]. Beijing: People's Medical Publishing House. 2011;49.

27. Boeck S, Jung A, Laubender RP, Neumann J, Egg R, Goritschan C, et al. EGFR pathway biomarkers in erlotinib-treated patients with advanced pancreatic cancer: translational results from the randomised, crossover phase 3 trial AIO-PK0104. British journal of cancer. 2013;108(2):469-76.

28. Lopez R, Mendez CM, Fernandez MJ, Reinoso CR, Aldana GQ, Fernandez MS, et al. Phase II trial of erlotinib plus capecitabine as first-line treatment for metastatic pancreatic cancer (XELTA study). Anticancer research. 2013;33(2):717-23.

29. Jeon EK, Won HS, Ko YH, Lee IS, Hong TH, You YK, et al. Comparison of the efficacy and the toxicity between gemcitabine with capecitabine (GC) and gemcitabine with erlotinib (GE) in unresectable pancreatic cancer. Journal of cancer research and clinical oncology. 2012;138(10):1625-30.

30. Heinemann V, Vehling-Kaiser U, Waldschmidt D, Kettner E, Märten A, Winkelmann C, et al. Gemcitabine plus erlotinib followed by capecitabine versus capecitabine plus erlotinib followed by gemcitabine in advanced pancreatic cancer: Final results of a randomised phase 3 trial of the 'arbeitsgemeinschaft internistische onkologie' (AIO-PK0104). Gut. 2013;62(5):751-9.

31. Fensterer H, Schade-Brittinger C, Muller HH, Tebbe S, Fass J, Lindig U, et al. Multicenter phase II trial to investigate safety and efficacy of gemcitabine combined with cetuximab as adjuvant therapy in pancreatic cancer (ATIP). Annals of oncology : official journal of the European Society for Medical Oncology. 2013;24(10):2576-81.

32. Merchan JR, Ferrell A, Macintyre J, Ciombor KK, Levi J, Ribeiro A, et al. Phase II study of gemcitabine, oxaliplatin, and cetuximab in advanced pancreatic cancer. American journal of clinical oncology. 2012;35(5):446-50.

33. Ko AH, Youssoufian $\mathrm{H}$, Gurtler J, Dicke $\mathrm{K}$, Kayaleh $\mathrm{O}$, Lenz HJ, et al. A phase II randomized study of cetuximab and bevacizumab alone or in combination with gemcitabine as first-line therapy for metastatic pancreatic adenocarcinoma. Investigational new drugs. 2012;30(4):1597-606. 
34. Esnaola NF, Chaudhary UB, O'Brien P, Garrett-Mayer E, Camp ER, Thomas $\mathrm{MB}$, et al. Phase 2 trial of induction gemcitabine, oxaliplatin, and cetuximab followed by selective capecitabine-based chemoradiation in patients with borderline resectable or unresectable locally advanced pancreatic cancer. International journal of radiation oncology, biology, physics. 2014;88(4):837-44.

35. Harder I, Ihorst G, Heinemann V, Hofheinz R, Moehler M, Buechler P, et al Multicentre phase II trial of trastuzumab and capecitabine in patients with HER2 overexpressing metastatic pancreatic cancer. British journal of cancer. 2012;106(6):1033-8.

36. Thomas G, Chardes T, Gaborit N, Mollevi C, Leconet W, Robert B, et al. HER3 as biomarker and therapeutic target in pancreatic cancer: new insights in pertuzumab therapy in preclinical models. Oncotarget. 2014;5(16):7138-48.

37. Safran H, Miner T, Bahary N, Whiting S, Lopez CD, Sun W, et al. Lapatinib and gemcitabine for metastatic pancreatic cancer. A phase II study. American journal of clinical oncology. 2011;34(1):50-2.

38. Wu Z, Gabrielson A, Hwang JJ, Pishvaian MJ, Weiner LM, Zhuang T, et al. Phase II study of lapatinib and capecitabine in second-line treatment for metastatic pancreatic cancer. Cancer chemotherapy and pharmacology. 2015;76(6):1309-14

39. Tiseo M, Boni L, Ambrosio F, Camerini A, Baldini E, Cinieri S, et al. Italian, Multicenter, Phase III, Randomized Study of Cisplatin Plus Etoposide With or Without Bevacizumab as First-Line Treatment in Extensive-Disease Small-Cell Lung Cancer: The GOIRC-AIFA FARM6PMFJM Trial. Journal of clinical oncology: official journal of the American Society of Clinical Oncology. 2017;35(12):1281-7

40. Olive KP, Jacobetz MA, Davidson CJ, Gopinathan A, McIntyre D, Honess D, et al. Inhibition of Hedgehog signaling enhances delivery of chemotherapy in a mouse model of pancreatic cancer. Science. 2009;324(5933):1457-61.

41. Sousa CM, Biancur DE, Wang X, Halbrook CJ, Sherman MH, Zhang L, et al. Pancreatic stellate cells support tumour metabolism through autophagic alanine secretion. Nature. 2016;536(7617):479-83.

42. Casanovas O, Hicklin DJ, Bergers G, Hanahan D. Drug resistance by evasion of antiangiogenic targeting of VEGF signaling in late-stage pancreatic islet tumors. Cancer Cell. 2005;8(4):299-309.

43. Xian X, Hakansson J, Stahlberg A, Lindblom P, Betsholtz C, Gerhardt H, et al. Pericytes limit tumor cell metastasis. The Journal of clinical investigation. 2006;116(3):642-51.

44. Reinmuth N, Liu W, Jung YD, Ahmad SA, Shaheen RM, Fan F, et al. Induction of VEGF in perivascular cells defines a potential paracrine mechanism for endothelial cell survival. FASEB J. 2001;15(7):1239-41.

45. Sahin IH, Iacobuzio-Donahue CA, O'Reilly EM. Molecular signature of pancreatic adenocarcinoma: an insight from genotype to phenotype and challenges for targeted therapy. Expert Opin Ther Targets. 2016;20(3):341-59.

46. Wang Y, Hu GF, Zhang QQ, Tang N, Guo J, Liu LY, et al. Efficacy and safety of gemcitabine plus erlotinib for locally advanced or metastatic pancreatic cancer: a systematic review and meta-analysis. Drug Des Devel Ther. 2016;10:1961-72.

47. Ciliberto D, Staropoli N, Chiellino S, Botta C, Tassone P, Tagliaferri P. Systematic review and meta-analysis on targeted therapy in advanced pancreatic cancer. Pancreatology: official journal of the International Association of Pancreatology (IAP) [et al]. 2016;16(2):249-58.

48. Brown PD, Giavazzi R. Matrix metalloproteinase inhibition: a review of anti-tumour activity. Annals of oncology: official journal of the European Society for Medical Oncology. 1995;6(10):967-74 